\title{
Isospin mixing within the symmetry restored density functional theory and beyond*
}

\author{
W. Satula ${ }^{1}$, J. DobacZewski ${ }^{1,2}$, M. KonieczkA ${ }^{1}$, \\ W. NAZAREWICZ ${ }^{1,3,4}$ \\ ${ }^{1}$ Faculty of Physics, University of Warsaw, ul. Hoża 69, PL-00-681 Warsaw, \\ Poland \\ ${ }^{2}$ Department of Physics, P.O. Box 35 (YFL), University of Jyväskylä, FI-40014 \\ Jyväskylä, Finland \\ ${ }^{3}$ Department of Physics and Astronomy, University of Tennessee, Knoxville, \\ Tennessee 37996, USA \\ ${ }^{4}$ Physics Division, Oak Ridge National Laboratory, Oak Ridge, Tennessee 37831, \\ USA
}

We present results of systematic calculations of the isospin-symmetrybreaking corrections to the superallowed $I=0^{+}, T=1 \rightarrow I=0^{+}, T=1 \beta$ decays, based on the self-consistent isospin- and angular-momentum-projected nuclear density functional theory (DFT). We discuss theoretical uncertainties of the formalism related to the basis truncation, parametrization of the underlying energy density functional, and ambiguities related to determination of Slater determinants in odd-odd nuclei. A generalization of the double-projected DFT model towards a no core shell-model-like configuration-mixing approach is formulated and implemented. We also discuss new opportunities in charge-symmetry- and charge-independencebreaking studies offered by the newly developed DFT formalism involving proton-neutron mixing in the particle-hole channel.

PACS numbers: $21.10 . \mathrm{Hw}, 21.60 . \mathrm{Jz}, 21.30 . \mathrm{Fe}$

\section{Introduction}

Isospin impurities in nuclear wave functions resulting from the isospinsymmetry non-conservation are of the order of a few percent. Still, they impact a plethora of nuclear phenomena, especially in self-conjugate nuclei. Of particular importance are the $\Delta T=0$ electric dipole transitions (E1)

* Presented at the XXXIII Mazurian Lakes Conference on Physics, Piaski, Poland, September 1-7, 2013 
in $N=Z$ nuclei, which, in the long-wavelength approximation, are isospin forbidden [1, 2, 3, and thus can proceed only via the isospin mixing. This offers a method that allows for an empirical assessment the isospin-symmetry breaking (ISB).

In case of the Fermi and Gamow-Teller $\beta$-decay transitions, the selection rules are $\Delta T=0$ and $\Delta T=0, \pm 1$, respectively, except for the $0^{+} \rightarrow 0^{+}$transitions that are pure Fermi decays [4]. Although the influence of the ISB corrections on $\beta$-decay rates is generally small, their precise knowledge is critically important for the superallowed Fermi $\beta$-decays $I=0^{+}, T=1 \rightarrow I=0^{+}, T=1$ between the isobaric analogue states (IAS) and, to a somewhat lesser degree, for the decays between the $T=1 / 2$ mirror nuclei. The reason is that these transitions provide the most precise data on the vector (Fermi) coupling constant $G_{V}$ and the leading element $V_{u d}$ of the Cabibbo-Kobayashi-Maskawa (CKM) flavour-mixing matrix. This allows for testing the unitarity of the CKM matrix, violation of which may indicate a new physics beyond the Standard Model of particle physics, see Ref. [5] and references quoted therein.

Because of the smallness of isospin impurities, their accurate microscopic calculation is a challenging task. The reason is that they result from a subtle balance between the isospin-symmetry conserving short-range strong interaction and the ISB long-range Coulomb interaction that polarizes the entire nucleus. Capturing that balance is possible only within the no core approaches, which, in heavier nuclei, reduces the possible choices to the nuclear DFT. The DFT, however, cannot be directly used to compute isospin impurities because of the unphysical isospin mixing caused by the spontaneous violation of isospin [6]. This obstacle hindered the progress in the field for decades. Only very recently, we have developed the isospin- and angular-momentum projected DFT approach capable of treating rigorously the (conserved) rotational symmetry and, at the same time, tackle the explicit breaking of the isospin symmetry due to the Coulomb field [7, $8,9,10]$.

The aim of this work is to present a brief overview of recent results for the isospin mixing and ISB corrections to the $I=0^{+}, T=1 \rightarrow I=0^{+}, T=1$ $\beta$-decays, focusing on theoretical uncertainties and limitations of the employed isospin- and angular-momentum-projected DFT framework. All calculations presented here were obtained by using the DFT solver HFODD version $(2.249 \mathrm{t})$ or higher [11, 12]. The paper is organized as follows. Basic features of the formalism are summarized in Sec. 2. Section 3 discusses the ISB corrections to superallowed $\beta$-decays, focusing on theoretical uncertainties. A dynamic variant of the model, which involvs the configuration mixing, is presented in Sec. 4 together with preliminary applications. Section 5 overviews new opportunities in studying the ISB mechanism offered by the newly developed proton-neutron-symmetry-breaking DFT. Finally, 
Sec. 6 summarizes the main findings of this work.

\section{Isospin and angular momentum projected DFT model}

The calculation starts with solving Skyrme-Hartree-Fock (SHF) equations without pairing using a Hamiltonian that consists the isospin-invariant kinetic energy and Skyrme interaction, and the Coulomb force being the only explicit source of isospin-symmetry violation in the model. The resulting self-consistent Slater determinant $|\varphi\rangle$ breaks rotational and isospin invariance. It is used as a reference to create a basis of good-isospin-goodangular-momentum states $\left|\varphi ; I M K ; T T_{z}\right\rangle$ :

$$
\left|\varphi ; I M K ; T T_{z}\right\rangle=\frac{1}{\sqrt{N_{\varphi ; I M K ; T T_{z}}}} \hat{P}_{T_{z}, T_{z}}^{T} \hat{P}_{M, K}^{I}|\varphi\rangle,
$$

where $\hat{P}_{T_{z}, T_{z}}^{T}$ and $\hat{P}_{M, K}^{I}$ stand for the standard isospin and angular-momentum projection operators 13, respectively. The Hamiltonian is then rediagonalized in the basis $\left|\varphi ; I M K ; T T_{z}\right\rangle$. Since the intrinsic quantum number $K$ is not conserved and the set (1) is overcomplete, the rediagonalization is done by first selecting the subset of linearly independent states, known as collective space [13]. For each $I$ and $T$ these states are spanned by the so-called natural states $\left|\varphi ; I M ; T T_{z}\right\rangle^{(i)}$ [14]. The resulting eigenfunctions are:

$$
\left|n ; \varphi ; I M ; T_{z}\right\rangle=\sum_{i, T \geq\left|T_{z}\right|} a_{i I T}^{(n ; \varphi)}\left|\varphi ; I M ; T T_{z}\right\rangle^{(i)},
$$

where index $n$ labels the eigenstates in ascending order of energies. The isospin impurities are defined as:

$$
\alpha_{\mathrm{C}}^{n}=1-\sum_{i}\left|a_{i I T}^{(n ; \varphi)}\right|^{2},
$$

where the sum is performed for a fixed value of the isospin $T$ that dominates the wave function (2). The impurities (3) are, by construction, free from the spurious isospin mixing.

The isospin impurities can be studied using the isospin-only-projected variant of the approach, which is free from singularities [7, 8, 9] plaguing angular-momentum or particle-number projections [15, 16, 17, 18, 19]. The calculated impurities are consistent with the recent data extracted from the giant-dipole-resonance decay studies in ${ }^{80} \mathrm{Zr}$ [20 and isospin-forbidden E1 decay in ${ }^{64} \mathrm{Ge}[21$. Both data points disagree with the standard mean-field (MF) results, which are lower by almost $30 \%$ due to spurious contaminations. This agreement shows that our model is capable of quantitatively capturing the magnitude of the isospin mixing, which is important in the 
context of applying it to the determination of the ISB corrections to the superallowed Fermi $\beta$-decay.

\section{Isospin-symmetry-breaking corrections to superallowed $\beta$-decay}

The projected DFT method allows for a rigorous quantum-mechanical calculation of the $I=0^{+}, T=1 \rightarrow I=0^{+}, T=1$ Fermi matrix element using the bare isospin operators $\hat{T}_{ \pm}$, that is,

$$
\left|\left\langle I=0, T \approx 1, T_{z}= \pm 1 ; \varphi\left|\hat{T}_{ \pm}\right| I=0, T \approx 1, T_{z}=0 ; \psi\right\rangle\right|^{2} \equiv 2\left(1-\delta_{\mathrm{C}}\right) .
$$

On the one hand, the state $\left|I=0, T \approx 1, T_{z}= \pm 1 ; \varphi\right\rangle$ is approximated by a double-projected state (2), where the self-consistent Slater determinant $|\varphi\rangle$ represents the ground state (g.s.) of even-even nucleus. The wave function $|\varphi\rangle$ is uniquely determined by occupying pairwise the deformed singleparticle (s.p.) orbitals from the bottom of a potential well up to the Fermi level. On the other hand, the double-projected state $\left|I=0, T \approx 1, T_{z}=0 ; \psi\right\rangle$ represents the anti-aligned exited state of the odd-odd $N=Z$ system. The anti-aligned configuration [10] is obtained by placing the odd neutron and odd proton in the lowest available time-reversed (or signature-reversed) s.p. orbitals $|\pi\rangle \otimes|\bar{\nu}\rangle$ (or $|\bar{\pi}\rangle \otimes|\nu\rangle$ ). Such arrangement manifestly breaks the isospin symmetry. Projecting out the $T=1$ component of the determinant $|\psi\rangle$ is essentially the only way of reaching the $|T \approx 1\rangle$ configurations in $N=Z$ nuclei. Indeed, these states are not at all representable by single Slater determinants built by occupying unmixed proton and neutron s.p. wave functions.

Calculation of matrix elements (4) requires both the isospin and angularmomentum projections [9, 10]. Since for density-dependent interactions the angular momentum projection is ill-defined [15, 18, the method can be used only for functionals originating from the true interaction. This is a severe limitation that eliminates all modern density-dependent Skyrme functionals and forces us to use the only available density-independent parametrization SV [23. Poor spectroscopic properties of SV result in uncertainties of individual $\delta_{\mathrm{C}}$ corrections. To assess these uncertainties, we developed the new density-independent Skyrme force SHZ2 by fitting its parameters to the selected bulk and s.p. properties of light doubly magic nuclei up to ${ }^{100} \mathrm{Sn}$, see Ref. [10. Data in light nuclei poorly constrain isovector properties of the force. As a consequence the symmetry energy of SHZ2 is $a_{\text {sym }}=42.2 \mathrm{MeV}$ exceeding the commonly acceptable value by almost $30 \%$. This property precludes applications of SHZ2 in nuclear structure studies, but it offers an opportunity to study the sensitivity of ISB corrections with respect to the 

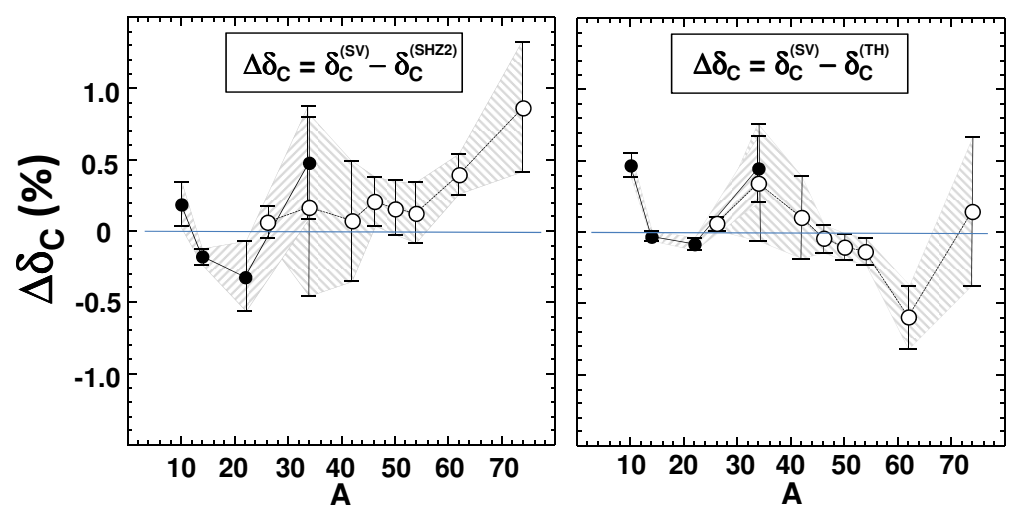

Fig. 1. Left: differences between the ISB corrections to the twelve accurately measured superallowed $0^{+} \rightarrow 0^{+} \beta$-transitions (excluding $A=38$ ) calculated using the double-projected DFT approach with the SV and SHZ2 functionals. Right: differences between the double-projected DFT results obtained with the SV functional and those of Ref. [22]. Circles and dots label the $T_{z}=-1 \rightarrow T_{z}=0$ and $T_{z}=0 \rightarrow T_{z}=1$ decays, respectively. Shaded bands mark the estimated theoretical errors.

symmetry energy, which governs response of the system against isovector distortions.

Differences between the ISB corrections calculated using the doubleprojected DFT with the SV and SHZ2 functionals are shown in Fig. 17. The error bars include two sources of theoretical uncertainties: $(i)$ the error due to the basis cutoff and (ii) the error resulting from averaging over the values obtained for different relative orientations of the nuclear shapes and currents associated with the valence neutron-proton pairs [10]. The error due to the basis size is rather conservatively estimated to be of the order of $\sim 10 \%$. It can be, in principle, reduced by taking a larger basis set. However, within the present formalism, ambiguities related to the shape and current orientations cannot be reduced further without taking into account the configuration mixing.

Surprisingly, the large difference between symmetry energies of SV and SHZ2 has a surprisingly modest impact on the calculated values of $\delta_{\mathrm{C}}$. Only for the heaviest nuclei considered, the values of $\delta_{\mathrm{C}}$ calculated using SHZ2 are somewhat reduced as compared to the SV results. The two sets of the calculated ISB corrections to the $I=0^{+}, T=1 \rightarrow I=0^{+}, T=1$ Fermi decay lead to $\left|V_{u d}\right|=0.97397(27)$ and $\left|V_{u d}\right|=0.97374(27)$, for SV and SHZ2, respectively [10]. Both values result in the unitarity of the CKM matrix up to $0.1 \%$ and both are fully consistent with the result obtained by Towner and Hardy (TH) 22] using different methodology based on the nuclear shellmodel combined with MF wave functions. It is gratifying to see that also 
individual SV values of $\delta_{\mathrm{C}}$ are consistent within $2 \sigma$ with the values calculated in Ref. [22], see Fig. 1 b. Two exceptions are the ISB corrections to ${ }^{10} \mathrm{C} \rightarrow{ }^{10} \mathrm{~B}$ and ${ }^{62} \mathrm{Ga} \rightarrow{ }^{62} \mathrm{Zn}$ transitions. Mutually consistent DFT and $\mathrm{TH}$ results are at variance with the RPA-based study of Ref. [24].

\section{Beyond multi-reference DFT}

As discussed in the previous section, the double-projected DFT model involves a single self-consistent Slater determinant, representing the ground state of an even-even $T_{z}= \pm 1$ nucleus, and a single Slater determinant $\psi$, representing the anti-aligned configuration in an odd-odd $T_{z}=0$ system. Owing to the ambiguities in choosing shape and current orientations, the latter configuration is not uniquely defined. To estimate associated uncertainties, one can averaging over results obtained for different reference states [10].

To overcome such difficulties, we have implemented an extended version of the model that allows for mixing of states projected from different self-consistent Slater determinants $\varphi_{i}$ representing low-lying (multi)particle(multi)hole excitations in a nucleus of interest. The extension can be viewed as a variant of the no core shell-model, with two-body effective interaction (including the Coulomb force) and a basis-truncation scheme dictated by the self-consistent deformed Hartree-Fock solutions. The scheme proceeds as follows:

- A set of low-lying (multi)particle-(multi)hole SHF states $\left\{\varphi_{i}\right\}$ is calculated along with their HF energies $e_{i}^{(H F)}$. These states form a basis of reference states for a subsequent projection.

- The projection techniques are applied to the set of states $\left\{\varphi_{i}\right\}$ to calculate a family $\left\{\Psi_{I}^{(\alpha)}, E_{I}^{(\alpha)}\right\}$ of good-angular momentum states with $K$-mixing and isospin mixing treated properly. The states $\left\{\Psi_{I}^{(\alpha)}\right\}$ are, in general, non-orthogonal.

- The mixing of states $\left\{\Psi_{I}^{(\alpha)}\right\}$ is performed by solving the Hill-Wheeler equation in the collective space spanned by the natural states corresponding to non-zero eigenvalues of the norm matrix, that is, by applying the same technique which is used to handle the $K$-mixing [14].

The model can be used to calculate spectra and transitions in any nucleus, irrespectively of its neutron- and proton-number parities. It can be also applied to compute $\beta$-decay matrix elements between different nuclei. In particular, it opens up a possibility of detailed studies of isovector sector of the underlying EDFs [25]. The numerical stability of the method is, however, affected by truncation errors. Namely, the numerically unstable 
Table 1. The ISB corrections $\delta_{\mathrm{C}}$ (in \%) in light nuclei corresponding to measured (top) and unmeasured (bottom) superallowed $\beta$-decays in selected parent nuclei. Shown are the results of dynamical calculations performed in this work, $\delta_{\mathrm{C}}^{(\mathrm{mix} ; \mathrm{SV})}$; double-projected DFT method with SV and SHZ2 obtained in Ref. [10; and the ISB corrections $\delta_{\mathrm{C}}^{(\mathrm{TH})}$ of Ref. [22].

\begin{tabular}{lrrrc} 
Nucleus & \multicolumn{1}{c}{$\delta_{\mathrm{C}}^{(\text {mix;SV })}$} & \multicolumn{1}{c}{$\delta_{\mathrm{C}}^{(\mathrm{SV})}$} & \multicolumn{1}{c}{$\delta_{\mathrm{C}}^{(\mathrm{SHZ} 2)}$} & $\delta_{\mathrm{C}}^{(\mathrm{TH})}$ \\
\hline$T_{z}=-1:$ & & & & \\
${ }^{10} \mathrm{C}$ & $0.668(67)$ & $0.65(14)$ & $0.462(65)$ & $0.175(17)$ \\
${ }^{14} \mathrm{O}$ & $0.303(30)$ & $0.303(30)$ & $0.480(48)$ & $0.330(25)$ \\
${ }^{22} \mathrm{Mg}$ & $0.268(54)$ & $0.301(87)$ & $0.342(49)$ & $0.380(22)$ \\
${ }^{34} \mathrm{Ar}$ & $0.87(17)$ & $1.11(29)$ & $1.08(42)$ & $0.665(56)$ \\
$T_{z}=0:$ & & & & \\
${ }^{26} \mathrm{Al}$ & $0.329(66)$ & $0.370(95)$ & $0.307(62)$ & $0.310(18)$ \\
${ }^{34} \mathrm{Cl}$ & $0.75(15)$ & $1.00(38)$ & $0.83(50)$ & $0.650(46)$ \\
\hline$T_{z}=-1:$ & & & & \\
${ }^{18} \mathrm{Ne}$ & $1.38(28)$ & $1.41(46)$ & $0.72(30)$ & $0.565(39)$ \\
${ }^{26} \mathrm{Si}$ & $0.427(85)$ & $0.47(10)$ & $0.529(77)$ & $0.435(27)$ \\
${ }^{30} \mathrm{~S}$ & $1.24(25)$ & $1.42(26)$ & $0.98(21)$ & $0.855(28)$ \\
$T_{z}=0:$ & & & & \\
${ }^{18} \mathrm{~F}$ & $1.22(24)$ & $1.25(42)$ & $0.42(24)$ & \\
${ }^{22} \mathrm{Na}$ & $0.257(26)$ & $0.35(14)$ & $0.216(86)$ & \\
${ }^{30} \mathrm{P}$ & $0.98(20)$ & $1.16(27)$ & $0.60(20)$ & \\
\hline \hline
\end{tabular}

solutions are removed the model space by truncating either the high-energy states $\left\{\Psi_{I}^{(\alpha)}\right\}$ or the natural states corresponding to small eigenvalues of the norm matrix, or by applying both truncations simultaneously. This procedure is not fully satisfactory, but it is relatively reliable for energy values. Estimated errors on stable eigenvalues rarely exceed $\pm 150 \mathrm{keV}$.

The source of the obtained instabilities is not fully recognized. They could be related to the zero range of the Skyrme force. Indeed, it is well known that the Dirac-delta force is unstable in three dimensions and requires regularization [26]. Work along these lines with finite-range densityindependent EDFs [27] is under way. Moreover, it is not clear whether EDFs or two-body interactions fitted at the MF level are realistic enough to be used in a beyond-multi-reference DFT theory.

At present, calculations can be performed only for the SV Skyrme interaction. First results communicated in Refs. 28, 25] are encouraging. Here, we present further applications of the formalism. Table 1 illustrates prelim- 
inary results for the ISB corrections in light nuclei for $A<42$ calculated using the extended model. In these calculations, we mixed states projected from the $\left|\psi^{(\mathrm{X})}\right\rangle,\left|\psi^{(\mathrm{Y})}\right\rangle$, and $\left|\psi^{(\mathrm{Z})}\right\rangle$ Slater determinants obtained in Ref. [10], which correspond to three different orientations of the s.p. alignment with respect to principal axes of the core. The results of such configurationmixing calculations are shown in the first column of the table, including the error bars that are estimated to be of the order of $10 \%$ for numerically stable solutions and $20 \%$ for cases requiring regularization. The new results are fully consistent with the average values quoted in Ref. [10].

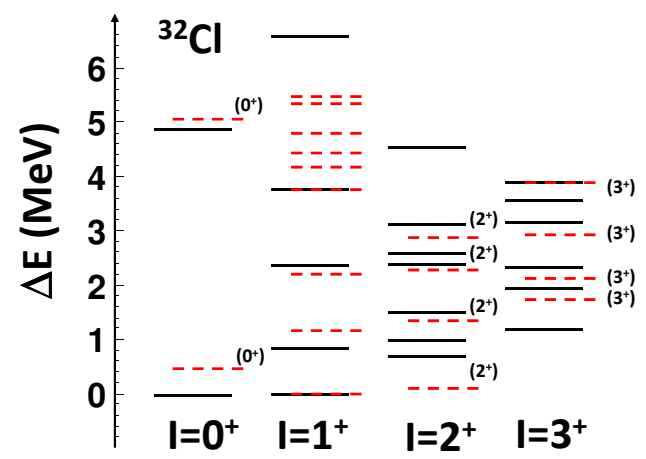

Fig. 2. Low-spin $I=0^{+}, 1^{+}, 2^{+}$, and $3^{+}$states in odd-odd nucleus ${ }^{32} \mathrm{Cl}$ plotted relative to the lowest $1^{+}$. Theoretical levels are marked by solid lines. Dashed lines indicate empirical data taken from Ref. 29. Note, that for all but $1^{+}$states, the empirical spin assignments are uncertain.

Recently, Melconian et al. [30] performed high precision measurement of the $\gamma$ yields following the $\beta$-decay of $I=1^{+}, T=1$ state in ${ }^{32} \mathrm{Cl}$ to its isobaric analogue state in ${ }^{32} \mathrm{~S}$ reporting for the Fermi branch an anomalously large value of $\delta_{\mathrm{C}} \approx 5.3(9) \%$. The physical reason for this enhancement can be traced back to a near-degeneracy of the $T=1$ isobaric analogue state at $7002 \mathrm{keV}$ and the $T=0$ state at $7190 \mathrm{keV}$ [29].

As discussed in Ref. [10, owing to ambiguities in choosing the reference Slater determinant, the static projected DFT approach is not sufficient to give a reliable prediction for $\delta_{\mathrm{C}}$. The case of ${ }^{32} \mathrm{Cl}$ provides an excellent playground for testing the dynamical variant of our model involving configuration mixing. This nucleus is a relatively weakly bound odd-odd system with tentative spin assignments for all but $1^{+}$states, see discussion in Ref. 29. Figure 2 compares calculated and empirical spectra of the low-spin $I=0^{+}$, $1^{+}, 2^{+}$, and $3^{+}$states in ${ }^{32} \mathrm{Cl}$. In our calculations, nine $1 \mathrm{p}-1 \mathrm{~h}$ configurations were considered. We see that the level of agreement is quite good and that the theory is capable of capturing the main features of experiment, in particular, the placement of $I=0^{+}$and $I=1^{+}$states. 
Similar dynamical calculations for ${ }^{32} \mathrm{~S}$ (with six $1 \mathrm{p}-1 \mathrm{~h}$ configurations included) indicate that the theory reproduces quite well the energy splitting between the isobaric analogue states $I=1^{+}, T=1$ and $I=1^{+}, T=0$ - critical for a reliable estimate of $\delta_{\mathrm{C}}$. As shown in Fig. 3 in our calculations the splitting is overestimated only by $\sim 160 \mathrm{keV}$ and total excitation energy of the isobaric analogue state is underestimated by $\sim 1.3 \mathrm{MeV}$. Furthermore, the theory well captures the ISB effects in the $I=1^{+}$states in ${ }^{32} \mathrm{~S}$ and ${ }^{32} \mathrm{Cl}$. Indeed, the calculated value of the ISB correction to the Fermi decay between the isobaric analogue states is $\delta_{\mathrm{C}} \approx 6(2) \%$. Large error bar accounts for the effect of regularization and for asymmetry in the number and structure of the $1 \mathrm{p}-1 \mathrm{~h}$ configurations included in dynamical calculations in ${ }^{32} \mathrm{~S}$ and ${ }^{32} \mathrm{Cl}$. We stress that our calculations contain no free parameters that can be readjusted to improve the agreement between the theory and experiment.

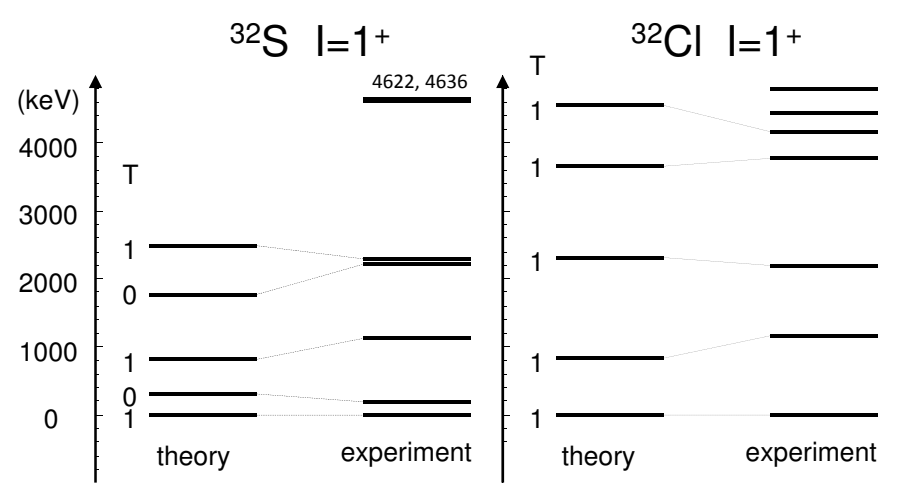

Fig. 3. Theoretical and empirical spin $1^{+}$states in ${ }^{32} \mathrm{~S}$ (left) and ${ }^{32} \mathrm{Cl}$ (right). The spectra in ${ }^{32} \mathrm{~S}$ are normalized to the isobaric analogue $1^{+}$state and consist of states above it. In the calculations six (nine) low-lying $1 \mathrm{p}-1 \mathrm{~h}$ configurations were taken into account for ${ }^{32} \mathrm{~S}\left({ }^{32} \mathrm{Cl}\right)$, respectively.

\section{DFT with broken neutron- and proton-number symmetries}

The nucleon-nucleon (NN) strong force $V_{12}^{N N}$ is dominated by the isospin-invariant part $\sim \alpha+\beta \vec{\tau}^{(1)} \cdot \vec{\tau}^{(2)}$. There exists, however, firm experimental evidence that the $\mathrm{NN}$ force also contains charge independence breaking (CIB) components $\sim \tau_{z}^{(1)} \tau_{z}^{(2)}$ and two types of charge symmetry breaking (CSB) components $\sim\left(\tau_{z}^{(1)}+\tau_{z}^{(2)}\right)$ (causing no isospin mixing) and $\sim \alpha\left(\tau_{z}^{(1)}-\tau_{z}^{(2)}\right)+\beta\left[\vec{\tau}^{(1)} \times \vec{\tau}^{(2)}\right]_{z}$ (producing isospin mixing) [31]. The experimental evidence for these terms comes, among the others, from differences in $\mathrm{nn}, \mathrm{pp}$ and $\mathrm{np}$ phase shifts and scattering lengths; differences in neutron/proton analyzing powers in np scattering; binding energy differences 
in mirror nuclei; and binding energy differences of isobaric analogue states.

The isospin structure of the NN force can be reexpressed in terms of the two-body spherical tensors in isospace including the isoscalar, isovector and isotensor components. In the DFT-rooted formalisms, which are not directly linked to the NN interaction, one often uses isoscalar functionals, which are bilinear (or higher order) in isoscalar and isovector one-body densities and currents [32, 8].

The dominant part of the ISB effect in the atomic nucleus is due to the Coulomb interaction. In fact, in most of the applications involving DFTrooted approaches, the Coulomb force is the only term violating the isospin symmetry. This is in spite of the perpetual problems in reproducing the binding-energy differences in mirror nuclei. This problem, known under the name of Nolen-Schiffer anomaly [33, is rather well studied, and it is generally agreed that its explanation requires CSB strong interaction [34, which appears to almost exactly cancel the Coulomb exchange contribution [35, 34].

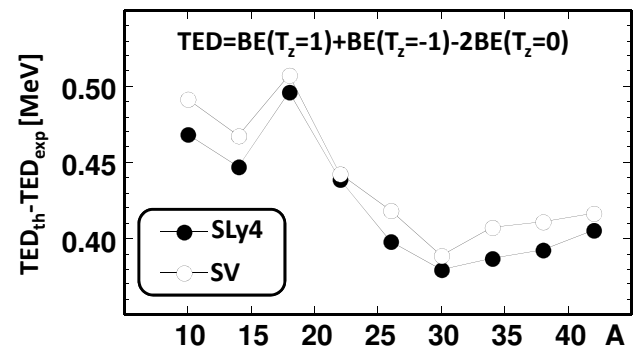

Fig. 4. Calculated triplet energy differences relative to experimental data. Filled and open circles mark calculations done with the SLy4 and SV EDFs, respectively.

The effect of CIB (or isotensor) interaction can be seen in triplet binding energy differences (TED) [36, which are defined as $\mathrm{TED}_{I} \equiv \mathrm{BE}_{I, T=1, T_{z}=-1}$ $+\mathrm{BE}_{I, T=1, T_{z}=+1}-2 \mathrm{BE}_{I, T=1, T_{z}=0}$. As mentioned in Sec. 3, the TEDs cannot be assessed by using the conventional MF, because the $\left|I, T=1, T_{z}=0\right\rangle$ states are not representable by a single Slater determinant built of unmixed proton and neutron states. Recently, we have extended the DFT formalism by breaking the neutron-proton symmetry at the particle-hole level [37. Therein, we have demonstrated that such a generalized DFT is capable of describing the isobaric analogue $\left|T=1, T_{z}=0\right\rangle$ states by evolving the $\left|T=1, T_{z}= \pm 1\right\rangle$ solutions by means of the tilted-axis cranking method in isospace. This will allow us to assess the effects of CSB and CIB strong interactions on ISB corrections to the superallowed $0^{+} \rightarrow 0^{+}$decays.

Results of our preliminary calculations for TEDs are shown in Fig. 4. Calculations were performed by using two different isospin-invariant Skyrme 
EDFs SV and SLy4 38. It is seen that the isotensor component of the Coulomb interaction is not strong enough to explain the experimental data. The systematic difference between the data and calculations can be viewed as an analog of the Nolen-Schiffer anomaly in mirror energy differences. The effect is, most likely, due to a missing CIB strong interaction components. It is interesting to observe that the unaccounted effect $\sim 450 \mathrm{keV}$ is almost $A$ - and Skyrme-force independent. Systematic studies of the TED anomaly are in progress.

\section{Summary and outlook}

The ISB corrections to the $0^{+} \rightarrow 0^{+}$superallowed Fermi $\beta$ decays obtained within the isospin- and angular-momentum projected DFT are critically overviewed. The dynamical extension of the model is proposed that promises to cure deficiencies of the previous approach by introducing configuration mixing. The dynamic model is applied, for the first time, to compute: $(i)$ the ISB corrections in light nuclei by mixing states projected of the Slater determinants in the odd-odd $N=Z$ nuclei obtained in Ref. [10; (ii) the low-spin spectra in ${ }^{32} \mathrm{Cl}$; (iii) the $I=1^{+}$states above the isobaric analogue state in ${ }^{32} \mathrm{~S}$; and $(i v)$ and the ISB correction to the ${ }^{32} \mathrm{Cl} \rightarrow{ }^{32} \mathrm{~S}$ Fermi $\beta$-decay branch between the isobaric analogue $I=1^{+}$states. In spite of outstanding problems related to the numerical stability of the method, our first results are encouraging. Indeed, our parameter-free calculations are able to capture the main empirical features. Finally, the Nolen-Schiffer anomaly for the isotensor CIB components of the NN interaction is formulated and discussed. Such advanced analysis was made possible by extending the standard nuclear DFT to the variant that includes the mixing of proton and neutron wave functions [28].

This work was supported in part by the U.S. Department of Energy under Contract No. DE-FG02-96ER40963 (University of Tennessee), No. DESC0008499 (NUCLEI SciDAC Collaboration), by the Academy of Finland and University of Jyväskylä within the FIDIPRO programme, and by the Polish National Science Center under Contract No. 2012/07/B/ST2/03907. We acknowledge the CSC-IT Center for Science Ltd, Finland for the allocation of computational resources.

\section{REFERENCES}

[1] L.E.H. Trainor, Phys. Rev. 85, 962 (1952).

[2] L.A. Radicati, Phys. Rev. 87, 521 (1952).

[3] W.M. MacDonald, Phys. Rev. 98, 60 (1955). 
[4] E.P. Wigner, Phys. Rev. 56, 519 (1939).

[5] I.S. Towner and J.C. Hardy, Rep. Prog. Phys. 73, 046301 (2010).

[6] C.A. Engelbrecht and R.H. Lemmer, Phys. Rev. Lett. 24, 607 (1970).

[7] W. Satuła et al., Phys. Rev. Lett. 103, 012502 (2009).

[8] W. Satuła et al., Phys. Rev. C 81, 054310 (2010).

[9] W. Satuła et al., Phys. Rev. Lett. 106, 132502 (2011).

[10] W. Satuła et al., Phys. Rev. C 86, 054314 (2012).

[11] N. Schunck et al., Comput. Phys. Commun. 183, 166 (2012).

[12] N. Schunck et al., unpublished.

[13] P. Ring and P. Schuck, The Nuclear Many-Body Problem (Springer-Verlag, Berlin, 1980).

[14] J. Dobaczewski et al., Comput. Phys. Commun. 180, 2361 (2009).

[15] M. Anguiano, J.L. Egido, and L.M. Robledo, Nucl. Phys. A696, 467 (2001).

[16] L.M. Robledo, Int. J. Mod. Phys. E 16, 337 (2007).

[17] J. Dobaczewski et al., Phys. Rev. C 76, 054315 (2007).

[18] H. Zduńczuk et al., Int. J. Mod. Phys. E 16, 377 (2007).

[19] D. Lacroix, T. Duguet, and M. Bender, Phys. Rev. C 79, 044318 (2009).

[20] A. Corsi et al., Acta Phys. Pol. B42, 619 (2011); Phys. Rev. C 84, 041304 (2011).

[21] E. Farnea et al., Phys. Lett. B551, 56 (2003).

[22] I.S. Towner and J.C. Hardy, Phys. Rev. C 77, 025501 (2008).

[23] M. Beiner et al., Nucl. Phys. A238, 29 (1975).

[24] H. Liang, N. Van Giai, and J. Meng, Phys. Rev. C 79, 064316 (2009).

[25] W. Nazarewicz et al., arXiv:1307:5782.

[26] C. Breit, Phys. Rev. 71, 215 (1947); C. Breit and P.R. Zilsel, Phys. Rev. 71, 232 (1947).

[27] K. Bennaceur, J. Dobaczewski, and F. Raimondi, arXiv:1305.7210.

[28] W. Satuła et al., arXiv:1307:1550.

[29] C. Ouellet and B. Singh, Nuclear Data Sheets 112, 2199 (2011).

[30] D. Melconian et al., Phys. Rev. Lett. 107, 182301 (2011).

[31] Isospin in nuclear physics, ed. D.H. Wilkinson (North Holland, Amsterdam, 1969).

[32] E. Perlińska et al., Phys. Rev. C69, 014316 (2004).

[33] J.A. Nolen and J.P. Schiffer, Ann. Rev. Nuc. Sci. 19, 471 (1969).

[34] B.A. Brown, W.A. Richter, and R. Lindsay, Phys. Lett. B483, 49 (2000).

[35] B.A. Brown, Phys. Rev. C58, 220 (1998).

[36] M.A. Bentley and S.M. Lenzi, Prog. Part. Nucl. Phys. 59, 497 (2007).

[37] K. Sato et al., arXiv:1308:1997.

[38] E. Chabanat et al., Nucl. Phys. A635, 231 (1998). 